\title{
On Gate Capacitance of Nanotube Networks
}

\author{
Zhiying Liu, Jiantong Li, Zhi-Jun Qiu, Zhi-Bin Zhang, Li-Rong Zheng, and Shi-Li Zhang
}

\begin{abstract}
This letter presents a systematic investigation of the gate capacitance $C_{G}$ of thin-film transistors (TFTs) based on randomly distributed single-walled carbon nanotubes (SWCNTs) in the channel. In order to reduce false counting of SWCNTs that do not contribute to current conduction, $C_{G}$ is directly measured on the TFTs using a well-established method for MOSFETs. Frequency dispersion of $C_{G}$ is observed, and it is found to depend on the percolation behavior in SWCNT networks. This dependence can be accounted for using an $R C$ transmission line model. These results are of important implications for the determination of carrier mobility in nanoparticle-based TFTs.
\end{abstract}

Index Terms-Frequency dependence, gate capacitance, nanotube networks, percolation, transmission line model.

\section{INTRODUCTION}

$\mathbf{T}$ HIN-FILM field-effect transistors (TFTs) featuring singlewalled carbon nanotube (SWCNT) networks as the semiconductor channel have gained enormous attention for large-area, inexpensive, and flexible electronic applications [1]-[3]. For TFTs, a reliable definition of the gate-to-channel capacitance $C_{G}$ is essential since it determines many key parameters such as drive current and carrier mobility. A reliable determination of $C_{G}$ requires proper understanding of the SWCNT network properties particularly SWCNT density

Manuscript received December 26, 2010; revised February 10, 2011; accepted February 11, 2011. Date of current version April 27, 2011. This work was supported in part by the Cultivation Fund of the Key Scientific and Technical Innovation Project, Ministry of Education of China, under Grant 708033, by the State Key Laboratory of ASIC and System at Fudan University, by the Chinese Scholarship Council, by the Swedish Research Council (VR) under Contract 2009-8068, by the Swedish Government Agency for Innovation Systems (VINNOVA) under Contract 2005-01138, and by the VINNOVA "iPack Vinnex Excellence Center." The review of this letter was arranged by Editor A. Ortiz-Conde.

Z. Liu is with the State Key Laboratory of ASIC and System, School of Microelectronics, Fudan University, Shanghai 200433, China, the Solid-State Electronics, The Ångström Laboratory, Uppsala University, 75121 Uppsala, Sweden, and also with iPack VINN Excellence Center, Royal Institute of Technology (KTH), 16440 Kista, Sweden.

$\mathrm{J}$. Li is with the School of Information and Communication Technology, Royal Institute of Technology (KTH), 16440 Kista, Sweden.

Z.-J. Qiu is with the State Key Laboratory of ASIC and System, School of Microelectronics, Fudan University, Shanghai 200433, China.

Z.-B. Zhang is with the Solid-State Electronics, The Ångström Laboratory, Uppsala University, 75121 Uppsala, Sweden and also with iPack VINN Excellence Center, Royal Institute of Technology (KTH), 16440 Kista, Sweden (e-mail: zhibin.zhang@angstrom.uu.se).

L.-R. Zheng is with the State Key Laboratory of ASIC and System, School of Microelectronics, Fudan University, Shanghai 200433, China, and also with iPack VINN Excellence Center, Royal Institute of Technology (KTH), 16440 Kista, Sweden.

S.-L. Zhang is with the Solid-State Electronics, The Ångström Laboratory, Uppsala University, 75121 Uppsala, Sweden and also with the State Key Laboratory of ASIC and System, School of Microelectronics, Fudan University, Shanghai 200433, China.

Color versions of one or more of the figures in this letter are available online at http://ieeexplore.ieee.org.

Digital Object Identifier 10.1109/LED.2011.2118733 and percolation behavior. A parallel-plate capacitor model, where $C_{G}$ is calculated using its simple relation to channel area and gate dielectric thickness, is often used as a simplification [1]. This simple treatment can lead to an erroneous $C_{G}$, in particular, for TFTs with low-density SWCNT networks [2], [4]. Capacitance measurement can be performed by depositing a metal layer on the SWCNT network forming a metal-insulator-semiconductor (MIS) capacitor structure [3]. Unfortunately, this method will likely lead to an overestimation of $C_{G}$ because it also includes SWCNTs that are present as isolated sticks having no contribution to current conduction in the channel [5], [6]. Apparently, a more reliable determination of $C_{G}$ needs to reduce such false SWCNT counts and focus on SWCNTs that actively participate in percolation. In this letter, we will employ the well-established $C_{G}$ measurement procedure for MOSFETs [7]. Specifically, capacitance measurement is directly performed on a TFT by grounding its source and drain terminals while sweeping the gate bias. By doing so, the isolated SWCNTs not electrically connected to the source (S) and drain (D) contact electrodes are effectively excluded. This approach has already been shown to be feasible for transistors with individual semiconducting SWCNTs [8]. In this letter, the $C_{G}$ for TFTs with different SWCNT coverage will be determined in order also to unveil under what circumstances a simple MIS structure may suffice. Strong frequency dispersion of $C_{G}$ is observed and can be well accounted for by using an $R C$ transmission line (TL) model [9].

\section{DEVICE FABRICATION AND CHARACTERIZATIONS}

Heavily doped Si wafers were used for device fabrication. After the growth of a 150-nm-thick $\mathrm{SiO}_{2}$ as the gate dielectric, the desired gate stack with a back-gate configuration was formed. The S and D electrodes consisted of a 40-nm-thick bilayer of Pd/Ti. Random SWCNT networks were formed by means of drop casting of SWCNT suspension onto the channel areas of length $L=200 \mu \mathrm{m}$ and width $W=100 \mu \mathrm{m}$; see Fig. 1(a). The SWCNT suspension consisted of pristine HiPco SWCNTs (1.1 nm in average diameter) dispersed in aqueous solution with 1-wt $\%$ sodium dodecylbenzene sulfonate. For each sample with a specific SWCNT suspension, the distribution, as also shown in Fig. 1(a), and, hence, the average density $\rho\left(\mu \mathrm{m}^{-2}\right)$ of SWCNTs were characterized by atomic force microscopy (AFM) at three randomly chosen locations. The average length $l_{\mathrm{NT}}$ of the SWCNTs was $\sim 1.5 \mu \mathrm{m}$. An SWCNT surface coverage $p=\rho l_{\mathrm{NT}}^{2}$ is defined to accommodate different nanotube lengths [6]. For the network in Fig. 1(a), $p=$ $6.5 \pm 2.0$ is obtained, and it is slightly above the percolation threshold $p_{c}=4.236^{2} / \pi \approx 5.7$ [5], [6]. Current-voltage $(I-V)$ measurements were performed on a Keithley 4200-SCS semiconductor analyzer. Capacitance-voltage $(C-V)$ measurements were conducted by superimposing a small ac signal $(30 \mathrm{mV}$ in amplitude) at different frequencies, $f_{m}$, to $V_{\mathrm{GS}}$. The bias 

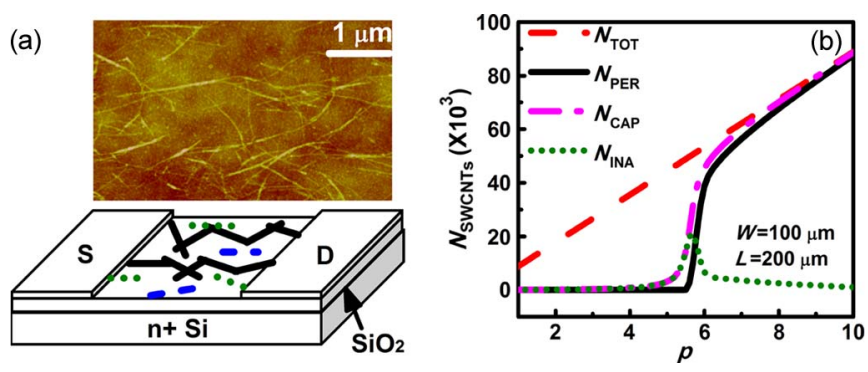

Fig. 1. (a) Schematic diagram of a back-gate TFT featuring randomly distributed SWCNTs consisting of (blue broken lines) isolated tubes, (red dotted lines) tubes nonpercolated but connected to the S/D electrodes or the percolation pathways, and (black solid lines) percolated tubes, together with an AFM image in height mode of an SWCNT network with surface coverage $p=6.5 \pm 2.0$. (b) Monte Carlo simulation results showing the variation of $N_{\mathrm{TOT}}, N_{\mathrm{PER}}, N_{\mathrm{CAP}}$, and $N_{\mathrm{INA}}$ (see corresponding text for their definition) with $p$ in a channel of $L=200 \mu \mathrm{m}$ and $W=100 \mu \mathrm{m}$. Each data point is obtained by averaging over 1000 independent Monte Carlo samples.

was applied using an Agilent 4284A $L C R$ meter to the gate electrode (i.e., Si substrate), while the $\mathrm{S}$ and $\mathrm{D}$ electrodes were both set to ground. Pulsed $V_{\mathrm{GS}}$ method was employed for both $C-V$ and $I-V$ measurements in order to effectively suppress the adverse effects of charge traps. The interval between two consecutive pulses was set to $10 \mathrm{~s}$ since the characteristic time for charge detrapping is $\sim 8 \mathrm{~s}$ [10]. All the electrical characterizations were carried out under ambient conditions.

\section{RESUlTS AND DiscUSSION}

The percolation behavior of SWCNT networks is depicted in Fig. 1(b). The results are obtained using Monte Carlo simulation [5] with 1.5- $\mu \mathrm{m}$-long sticks randomly distributed in a channel region $L=200 \mu \mathrm{m}$ long and $W=100 \mu \mathrm{m}$ wide. As expected, the total number of SWCNTs $N_{\text {TOT }}$ linearly increases with $p$. The number of SWCNTs forming percolation paths and, hence, carrying current between $\mathrm{S}$ and $\mathrm{D}, N_{\mathrm{PER}}$, rises sharply from zero when $p$ is greater than $p_{c}$. These SWCNTs are active for conducting current as well as for contributing to $C_{G}$. There is another class of SWCNTs, denoted $N_{\text {INA, that }}$ do not percolate but are still connected to the S/D electrodes or the percolation pathways. These SWCNTs contribute to $C_{G}$ but not to current conduction. They are slightly most populous below $p_{c}$, but overall, they only constitute a rather insignificant fraction of $N_{\text {TOT }}$. Thus, $C_{G}$ presents a good approximation of the capacitance corresponding to current conduction since the sum of $N_{\mathrm{PER}}$ and $N_{\mathrm{INA}}$, yielding $N_{\mathrm{CAP}}$, determines $C_{G}$, and the development of $N_{\mathrm{PER}}$ with $p$ indeed closely follows that for $N_{\mathrm{CAP}}$ in Fig. 1(b). For $p$ near and below $p_{c}, N_{\mathrm{CAP}}$ and $N_{\mathrm{PER}}$ differ significantly from $N_{\text {TOT }}$. Most of the SWCNTs remain electrically inactive. The difference between $N_{\mathrm{CAP}} / N_{\mathrm{PER}}$ and $N_{\text {TOT }}$ rapidly disappears with increasing $p$ above $p_{c}$, and now, the majority of SWCNTs resides in the percolating pathways.

The transfer characteristics of SWCNT-network TFTs with different $p$ measured by sweeping the gate bias $V_{\mathrm{GS}}$ from +40 to $-40 \mathrm{~V}$ at a fixed drain bias $V_{\mathrm{DS}}=-1 \mathrm{~V}$ are shown in Fig. 2(a). A nonpercolated network with $p=1.8$ gives rise to no conduction current, i.e., $I_{\mathrm{DS}}$ at noise level. For TFTs with percolated networks, the ON-state resistance $R_{\mathrm{ON}}$ measured at $V_{\mathrm{GS}}=-40 \mathrm{~V}$ and the ON/OFF current ratio, $I_{\mathrm{ON}} / I_{\mathrm{OFF}}$, decrease with increasing $p$, cf. Fig. 2(b). For the SWCNT densities of interest here, the intertube resistance predominates,
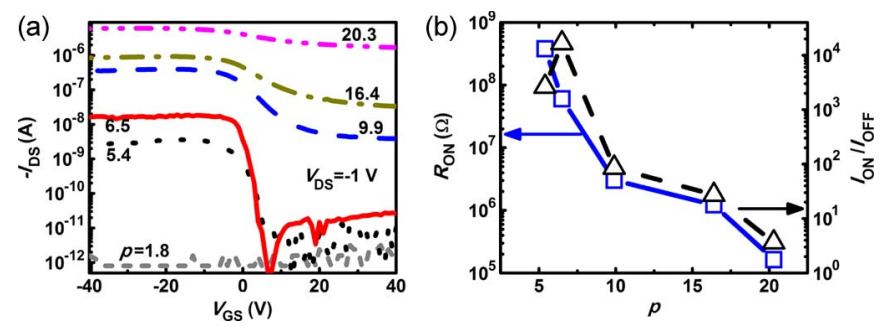

Fig. 2. (a) Transfer characteristics of SWCNT-network TFTs at different $p$ with pulsed $V_{\mathrm{GS}}$ sweeping from +40 to $-40 \mathrm{~V}$ and $V_{\mathrm{DS}}=-1 \mathrm{~V}$, and (b) (Blue squares) $R_{\mathrm{ON}}$ (at $V_{\mathrm{GS}}=-40 \mathrm{~V}$ ) and (black triangles) $I_{\mathrm{ON}} / I_{\mathrm{OFF}}$ as a function of $p$.

and the contact resistance between the SWCNTs and the metal electrodes is of less importance [11]. By referring to the different percolation domains of SWCNT networks [5], the strong dependence of $R_{\mathrm{ON}}$ on $p$ reflects the percolation transport in the network, and the large $I_{\mathrm{ON}} / I_{\mathrm{OFF}}$ near $p_{c}$ likely implies the formation of mixed percolation paths by both metallic $(m-)$ and semiconducting $(s-)$ SWCNTs or those by $s$-SWCNTs alone. The rapid reduction of $R_{\mathrm{ON}}$ and $I_{\mathrm{ON}} / I_{\mathrm{OFF}}$ at and above $p=9.9$ is likely caused by the onset of $m$-SWCNT percolation [5], [12], [13].

The employment of pulsed- $V_{\mathrm{GS}} C-V$ measurement assures an identical flatband voltage for TFTs with different $p$ due to the suppression of adverse effects of charge traps. For TFTs with low-density networks, e.g., $p=1.8$, the total capacitance measured is, within resolution limits, identical to that of the S/D contact electrodes alone $(p=0)$. For a percolated film with $p \geq$ $p_{c}$, a net capacitance becomes determinable by subtracting the contribution of the S/D electrodes from the total capacitance.

After removal of the capacitance contribution of the S/D electrodes, the $C-V$ curves measured with $V_{\mathrm{GS}}$ from +40 to $-40 \mathrm{~V}$ and at different $f_{m}$ are shown in Fig. 3(a) for the TFT with $p=6.5$. When $V_{\mathrm{GS}}$ is negative, holes are accumulated, and the capacitance stays at a high plateau. The capacitance drops when $V_{\mathrm{GS}}$ moves toward the positive direction. Normally, the capacitance in depletion is about $1 / 3$ of that in accumulation for SWCNT networks on a 30-nm-thick $\mathrm{SiO}_{2}$ gate dielectric layer [14]. A thicker $\mathrm{SiO}_{2}$ as used in this letter $(150 \mathrm{~nm})$ would result in a smaller capacitance difference. However, the capacitance difference is much larger, and in depletion, it is only about $1 / 10$ of that in accumulation in our case. This behavior cannot be explained by considering the model with a series connection of $C_{G}$ with the nanotube quantum capacitance [1] and is instead attributed to the TL effect to be discussed hereinafter. It is noticed, however, that the capacitance does not increase at positive $V_{\mathrm{GS}}$ at which inversion in the channel should occur. Such an increase is expected for an ordinary MOSFET [7] and has been shown for TFTs with individual SWCNTs [8]. The high-electron Schottky barrier between the $\mathrm{Pd}$ electrodes and the SWCNTs is, hence, suggested to be the hindrance for electron injection into the SWCNT-network channel. This interpretation is supported by the unipolar $p$-type transfer characteristics of such TFTs as shown in Fig. 2(a).

The accumulation capacitance $C_{A}$ measured at $V_{\mathrm{GS}}=$ $-40 \mathrm{~V}$ for the TFT with $p=6.5$ stays around $2.2 \mathrm{pF}$ when $f_{m}$ is below $2 \mathrm{kHz}$. It decreases continuously and quickly with increasing $f_{m}$. Such a strong frequency dependence of $C_{A}$ is observed for all TFTs with different $p$ as shown in Fig. 3(b). It is clearly seen that $C_{A}$ stays constant at low $f_{m}$ 

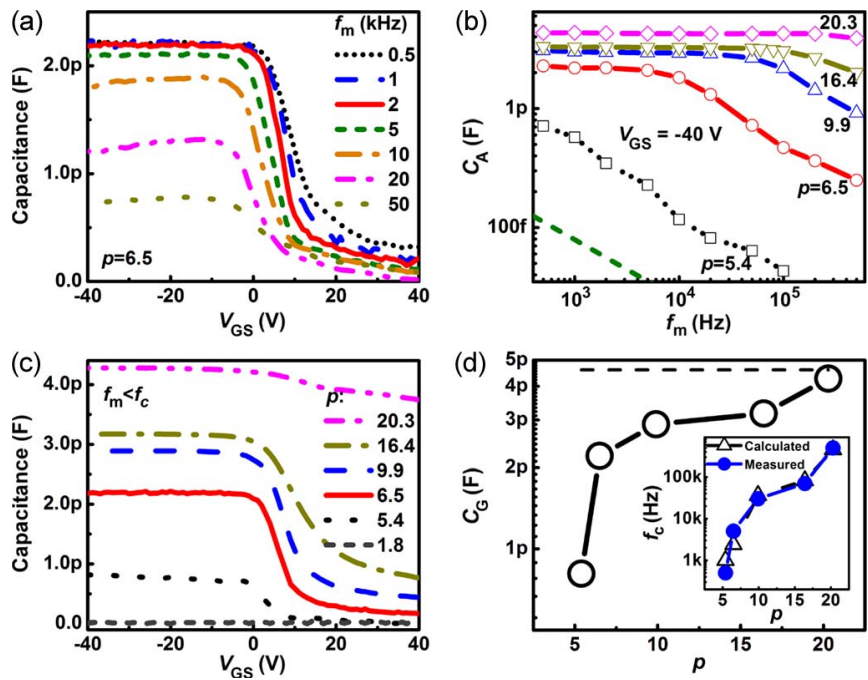

Fig. 3. (a) $C-V$ curves for the TFT with $p=6.5$ at different $f_{m}$ with pulsed $V_{\mathrm{GS}}$ from +40 to $-40 \mathrm{~V}$. (b) Frequency dependence of accumulation capacitance $C_{A}$ extracted at $V_{\mathrm{GS}}=-40 \mathrm{~V}$ for five TFTs with different $p$. The green dashed line has a slope of -0.5 . (c) $C-V$ curves for the TFTs with different $p$ at $f_{m}<f_{c}$ so that the TL effect is absent in accumulation. (d) Measured $C_{G}$ in accumulation obtained at $V_{\mathrm{GS}}=-40 \mathrm{~V}$ in (c), in comparison to (horizontal dashed line) the parallel-plate capacitance $C_{P}$. Inset: Excellent agreement between (black open triangles) calculated and (blue solid circles) measured $f_{c}$.

and starts to drop (i.e., occurrence of frequency dispersion) over a critical frequency $f_{c}$. The results in Fig. 3(b) further indicate that $f_{c}$ increases with increasing $p$. For $p \sim 6.5$ at which the percolation is likely to be dominated by $s$ - and $m$ SWCNTs in mixed mode or $s$-SWCNTs alone, $f_{c}$ is $\sim 5 \mathrm{kHz}$. Once $m$-SWCNTs alone starts to percolate at high $p, f_{c}$ quickly increases to tens and even hundreds of kilohertz.

To quantitatively analyze the dispersion, the SWCNT networks were treated using an $R C$ TL model with $R$ as the dc resistance and $C$ as the electrostatic capacitance [9]. The discrete elements over the gate length $(L)$ are the resistance per length $r=R / L$ and the capacitance per length $c=C / L$. From the basic TL theory, the attenuation constant can be expressed as $\alpha=$ $\left(\pi f_{m} r c\right)^{1 / 2}$. Thus, the charging voltage along the tubes drops exponentially with distance at a rate determined by $\alpha$. By letting the characteristic delay length $1 / \alpha$ equal to $L, f_{c}=1 /(\pi R C)$ is obtained. Above $f_{c}$, the channel charging effects cannot be neglected. The log-log plot in Fig. 3(b) shows that the slope of $C_{A}$ above $f_{c}$ is indeed $\sim-0.5$, i.e., $C \propto f_{m}^{-1 / 2}$, confirming the validity of the TL model. It is, therefore, straightforward to expect that the influence of TL effects on the capacitance in depletion will be much more profound due to a higher channel resistance. As a result, a substantial capacitance difference between accumulation and depletion is observed in Fig. 3(a).

By appropriately choosing $f_{m}$ in order to assure $f_{m}<f_{c}$ so that the channel charging is sufficiently fast, the $C-V$ curves for the TFTs with different $p$ were obtained and are shown in Fig. 3(c). The $C-V$ curves shift downward with decreasing $p$, as expected. As shown in Fig. 3(d), the measured $C_{G}$, which is currently in the absence of TL effects, is considerably smaller than what the parallel-plate capacitor model would predict, i.e., $C_{P}$. In consistency with the reported simulation results [2], [4], $C_{A}$ increases continuously and approaches $C_{P}$ with increasing $p$. The effective area of the SWCNT networks can be estimated by the ratio $C_{G} / C_{P}$. For a sufficiently dense film, $C_{G} / C_{P}$ is close to one, and the extracted mobility via $C_{P}$ should, thus, be close to that obtained by using an MIS structure [3]. For a film with $p$ around $p_{c}, C_{G} / C_{P}<1$ and, consequently, the extracted mobility via $C_{P}$ would be severely underestimated. For TFTs with percolated SWCNT networks, $f_{c}$ is calculated by employing the $R C$ TL model with the measured $R_{\mathrm{ON}}$ and $C_{G}$. As shown in the inset of Fig. 3(d), the calculated $f_{c}$ (black open triangles) coincides well with its measurement counterpart (blue solid circles). The result once again corroborates the validity of the TL model for TFTs based on SWCNT networks. It is, therefore, critical to obtain capacitance values in the absence of channel charging effects.

\section{CONCLUSion}

We have systematically investigated the $C_{G}$ of SWCNTnetwork-based TFTs. By employing a well-established characterization method for MOSFETs, it is found that the SWCNTs responsible for current conduction constitute the absolute majority of those contributing to $C_{G}$. A strong correlation between SWCNT density and $C_{G}$ is established with an anticipated low $C_{G}$ for low density. We have further studied the frequency dispersion of $C_{G}$ by employing the $R C$ TL model. Measurement of $C_{G}$ in the absence of channel charging effects has then been achieved, which is crucial for a reliable extraction of carrier mobility. These results can provide useful guidelines for nanoparticle-based TFTs.

\section{REFERENCES}

[1] E. Artukovic, M. Kaempgen, D. Hecht, S. Roth, and G. Gruner, "Transparent and flexible carbon nanotube transistors," Nano Lett., 5, no. 4, pp. 757-760, Mar. 2005.

[2] E. Snow, P. Campbell, M. Ancona, and J. Novak, "High mobility carbonnanotube thin-film transistors on a polymeric substrate," Appl. Phys. Lett., vol. 86, no. 3, p. 033105 , Jan. 2005.

[3] Q. Cao, H. Kim, N. Pimparkar, J. Kulkarni, C. Wang, M. Shim, K. Roy, M. Alam, and J. Rogers, "Medium scale carbon nanotube thin film integrated circuits on flexible plastic substrates," Nature, vol. 454, no. 7203, pp. 495-500, Jul. 2008.

[4] J. Guo, S. Goasguen, M. Lundstrom, and S. Datta, "Metal-insulatorsemiconductor electrostatics of carbon nanotubes," Appl. Phys. Lett., vol. 88, no. 8, pp. 1486-1488, Aug. 2002.

[5] J. Li, Z.-B. Zhang, and S.-L. Zhang, "Percolation in random networks of heterogeneous nanotubes," Appl. Phys. Lett., vol. 91, no. 25, p. 253127 , Dec. 2007.

[6] C. Kocabas, N. Pimparkar, O. Yesilyurt, S. Kang, M. Alam, and J. Rogers, "Experimental and theoretical studies of transport through large scale, partially aligned arrays of single-walled carbon nanotubes in thin film type transistors," Nano Lett., vol. 7, no. 5, pp. 1195-1202, Mar. 2007.

[7] D. K. Schroder, Semiconductor Material and Device Characterization. New York: Wiley-IEEE Press, 2006, pp. 371-372.

[8] S. Ilani, L. Donev, M. Kindermann, and P. McEuen, "Measurement of the quantum capacitance of interacting electrons in carbon nanotubes," Nat. Phys., vol. 2, no. 10, pp. 687-691, Oct. 2006.

[9] G. Esen, M. Fuhrer, M. Ishigami, and E. Williams, "Transmission line impedance of carbon nanotube thin films for chemical sensing," Appl. Phys. Lett., vol. 90, no. 12, p. 123 510, Mar. 2007.

[10] M. Qu, Z.-J. Qiu, Z.-B. Zhang, H. Li, J. Li, and S.-L. Zhang, "Chargeinjection-induced time decay in carbon nanotube network-based FETs," IEEE Electron Device Lett., vol. 31, no. 10, pp. 1098-1100, Dec. 2010.

[11] J. Li, Z.-B. Zhang, Z.-J. Qiu, and S.-L. Zhang, "Contact-electrode insensitive rectifiers based on carbon nanotube network transistors," IEEE Electron Device Lett., vol. 29, no. 5, pp. 500-502, Apr. 2008.

[12] E. Snow, P. Novak, P. Campbell, and D. Park, "Random networks of carbon nanotubes as an electronics material," Appl. Phys. Lett., vol. 82, no. 13, pp. 2145-2147, Mar. 2003.

[13] S. Kumar, N. Pimparkar, J. Murthy, and M. Alam, "Theory of transfer characteristics of nanotube network transistors," Appl. Phys. Lett., vol. 88, no. 12, p. 123 505, Mar. 2006.

[14] E. Snow and F. Perkins, "Capacitance and conductance of single-walled carbon nanotubes in the presence of chemical vapors," Nano Lett., vol. 5 , no. 12 , pp. $2414-2417$, Oct. 2005. 